\section{Assessing the short-term effects of radiotherapy on the shear modulus of the common carotid artery as a new biomarker of radiation-induced atherosclerosis}

\author{
Alireza Mohammadkarim ${ }^{1 *}$, Manijhe Mokhtari-Dizaji' , Ali Kazemian², Hazhir Saberi', \\ Niloofar Ayoobi Yazdi ${ }^{3}$, Mahbod Esfehani ${ }^{4}$ \\ 'Department of Medical Physics, Faculty of Medical Sciences, Tarbiat Modares University, \\ Tehran; ${ }^{2}$ Radiation Oncology Research Center, Cancer Institute, Tehran University of Medical \\ Sciences, Tehran; ${ }^{3}$ Department of Radiology, Imam Khomeini Hospital, Tehran University \\ of Medical Sciences, Tehran; ${ }^{4}$ Department of Radiation Oncology, Cancer Institute of Iran, \\ Tehran University of Medical Sciences, Tehran, Iran
}

Purpose: The purpose of this study was to investigate the incidence of short-term atherosclerosis in the common carotid arteries following radiotherapy.

Methods: The mean radiation dose to the arteries was $49.30 \pm 15.83$ Gy. A computational ultrasound method was introduced to investigate the elastic modulus. Ultrasonography was performed 2-3 cm inferior to the bifurcation region before and after radiotherapy, and sequential images were extracted from a video of each artery. Instantaneous movement of the arterial wall in the radial and longitudinal directions was extracted by implementing the maximum gradient and block matching algorithms, respectively.

Results: There was a significant change in systolic blood pressure after radiotherapy ( $P=0.008)$. Irradiated arteries had significantly smaller systolic and end-diastolic diameters than nonirradiated arteries $(P<0.001)$. The shear modulus was significantly different between irradiated and non-irradiated arteries $(3.10 \pm 2.03 \mathrm{kPa}$ vs. $1.38 \pm 0.98 \mathrm{kPa}, \mathrm{P}<0.001)$. The shear and Young moduli of radiation-induced arteries were $2.25 \pm 1.50$ and $1.57 \pm 0.59$ times higher than those of the pre-irradiation arteries.

Conclusion: The arterial shear modulus can be considered as a new biomarker of radiationinduced atherosclerosis in the common carotid artery.

Keywords: Ultrasonography; Carotid artery; Image processing; External radiotherapy;

Shear modulus

Key points: Stiffness of the common carotid artery increases following neck region radiotherapy as an early side effect of irradiation. Shear modulus derived by ultrasonography is a new biomarker of radiation-induced atherosclerosis. Shear modulus is more sensitive than the Young modulus in the diagnosis of early effects of radiotherapy for arterial damages.

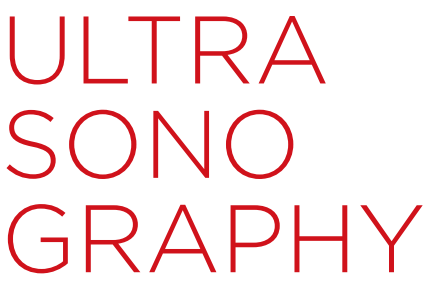

ORIGINAL ARTICLE

https://doi.org/10.14366/usg.21032 pISSN: 2288-5919 - elSSN: 2288-5943

Ultrasonography 2022;41:114-123

Received: February 13, 2021

Revised: April 23, 2021

Accepted: April 26, 2021

Correspondence to:

Manijhe Mokhtari-Dizaji, PhD, Department of Medical Physics, Faculty of Medical Sciences, Tarbiat Modares University, Tehran 1411713116, Iran

Tel. +98-21-82883893

Fax. +98-21-88006544

E-mail:mokhtarm@modares.ac.ir

${ }^{*}$ Current affiliation: Department of Medical Physics, Faculty of Medicine, Semnan University of Medical Sciences, Semnan, Iran

This is an Open Access article distributed under the terms of the Creative Commons Attribution NonCommercial License (http://creativecommons.org/ licenses/by-nc/4.0/) which permits unrestricted noncommercial use, distribution, and reproduction in any medium, provided the original work is properly cited.

Copyright @ 2022 Korean Society of Ultrasound in Medicine (KSUM)

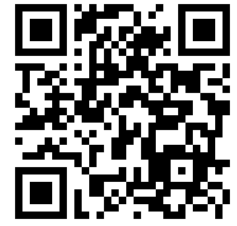

How to cite this article:

Mohammadkarim A, Mokhtari-Dizaji M Kazemian A, Saberi H, Yazdi NA, Esfehani M. Assessing the short-term effects of radiotherapy on the shear modulus of the common carotid artery as a new biomarker of radiation-induced atherosclerosis. Ultrasonography. 2022 Jan;41(1):114-123. 


\section{Introduction}

External radiotherapy is a common treatment for many head and neck malignancies [1-3]. Radiation-induced carotid atherosclerosis is a major late complication reported after external radiotherapy and occurs in $75 \%$ of cases [4]. Several recent studies have demonstrated that the intima-media thickness (IMT) changes as an early biomarker of radiation-induced atherosclerosis [5-8]. It has also been reported that radiation-induced damage of the common carotid arteries resulted in vascular stenosis $[1,2,9]$. Despite all the above, unfortunately, the carotid artery is not considered a high-risk organ that should be avoided in the treatment of the neck area [6]. Furthermore, due to the structure of the carotid artery in the neck, new treatment design techniques inevitably deliver radiation to this artery. Thus, even today, with the treatment of cancers of the head and neck using intensity-modulated radiation therapy (IMRT), the carotid artery receives a significant and non-theoretical radiation dose, and the use of the IMRT treatment technique only leads to a dose reduction of about $10 \mathrm{~Gy}$ [4].

Healthy and abnormal tissues show different elastic behavior due to changes in tissue density. Therefore, by measuring tissue elasticity, normal and abnormal tissues can be distinguished from each other. In addition, the hemodynamic characteristics of radiation-induced carotid arteries, including the mean velocity of blood, systolic to diastolic velocity ratio, and blood volume flow, are reliable indicators of the severity of atherosclerotic injury [10].

The main goal of the present study is to introduce a novel noninvasive ultrasonographic method for testing the Young and shear elastic moduli of the common carotid arteries in patients whose arteries have received considerable radiation doses. Therefore, patients treated using three-dimensional conformal techniques were selected in this study, and patients treated with more modern techniques were excluded. It is noteworthy that the mean dose of the carotid artery must be limited to $70 \mathrm{~Gy}$, as it is an at-risk organ [11], and dose delivery was done with consideration of the permissible range of doses based on the related therapeutic plans. At this stage, the received dose of patients' common carotid artery was extracted based on the appropriate treatment design program. Patterns of the biomechanical behavior of the carotid artery as a high-risk organ that is often neglected in clinical applications were presented. To confirm the reliability of the arterial shear elastic modulus as a tool for the diagnosis of radiation-induced carotid atherosclerosis, other well-known ultrasonographic biomarkers such as the Young elastic modulus and IMT were examined in each case. Differences in the arterial elastic behavior in the shear and radial directions were also compared as part of the data analysis.

\section{Materials and Methods}

\section{Compliance with Ethical Standards}

All procedures performed in studies involving human participants were in accordance with the ethical standards of the ethical committee of Tarbiat Modares University (6570.1393.REC.TMU. IR), which approved the protocol of the study, and with the 1964 Declaration of Helsinki and its later amendments or comparable ethical standards. Informed consent was obtained from all individual participants included in the study.

\section{Study Population}

A total of 24 consecutive head and neck carcinoma patients $(57 \pm 15$ years; body mass index, $25 \pm 5 \mathrm{~kg} / \mathrm{m}^{2} ; 15$ men and 9 women) treated with external radiotherapy were studied before and after external radiotherapy (Imam Khomeini Hospital, Tehran, Iran). The procedures performed in the present study were approved by the Medical Ethics Committee of Tarbiat Modares University and were conducted in accordance with the 1964 Declaration of Helsinki. Informed consent was obtained from all individual participants included in the study. The radiation therapy procedure was carried out with a high-energy linear accelerator using 6 MV photon beams delivering 2 Gy per session over 5 consecutive days per week for 6 weeks (Varian Medical Systems, Palo Alto, CA, USA). The participants were adults. None of them had a history of diabetes, stroke, or vascular surgery. Patients' resting blood pressure was recorded prior to each test. The tumors were in the oral cavity (38\%), larynx (33\%), parotid gland $(8 \%)$, lymph tissue $(8 \%)$, oropharyngeal region $(1 \%)$, glomus $(1 \%)$, and thyroid (1\%). Nine patients had a history of chemotherapy, five had a history of smoking, four had a history of various vascular diseases, and three had hypercholesterolemia. According to the medical history form of the patients referred for radiation therapy, one patient had a benign tumor and 23 patients underwent radiation therapy due to a malignancy. Eight of those tumors were stage 1 , four were stage 2 , five were stage 3 , and six were stage 4 .

\section{Treatment Planning}

All patients were treated with directly planned fields of radiotherapy using a single isocenter. In order to determine the dose of the carotid artery in each patient, consecutive computed tomography scan images were examined. Using the RT Dose treatment design software (Madison, WI, USA), the patients' common carotid artery was contoured from $2-3 \mathrm{~cm}$ before the carotid artery bifurcated to the end of the common carotid artery (Fig. 1A). Finally, a threedimensional volume of the common carotid artery was obtained (Fig. 1B). Then, based on the dose-volume histogram curve and the area under the curve (Fig. 1C), the mean dose that the carotid artery 


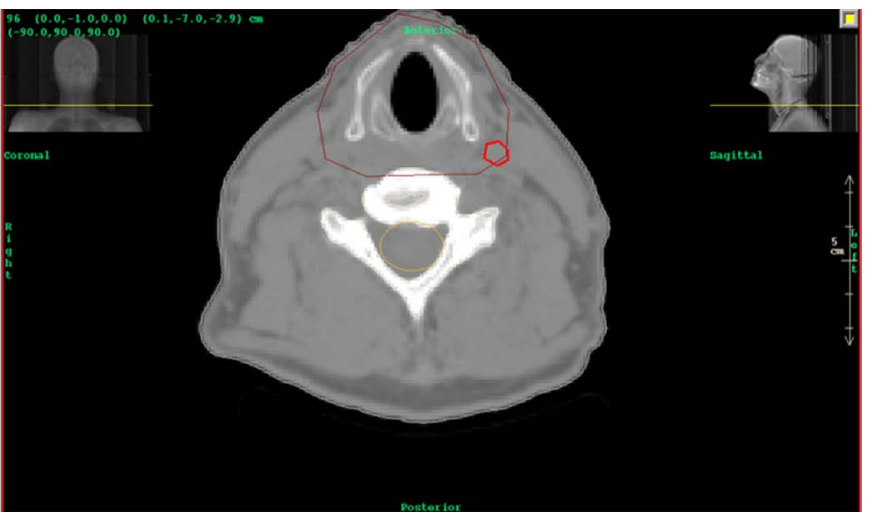

A

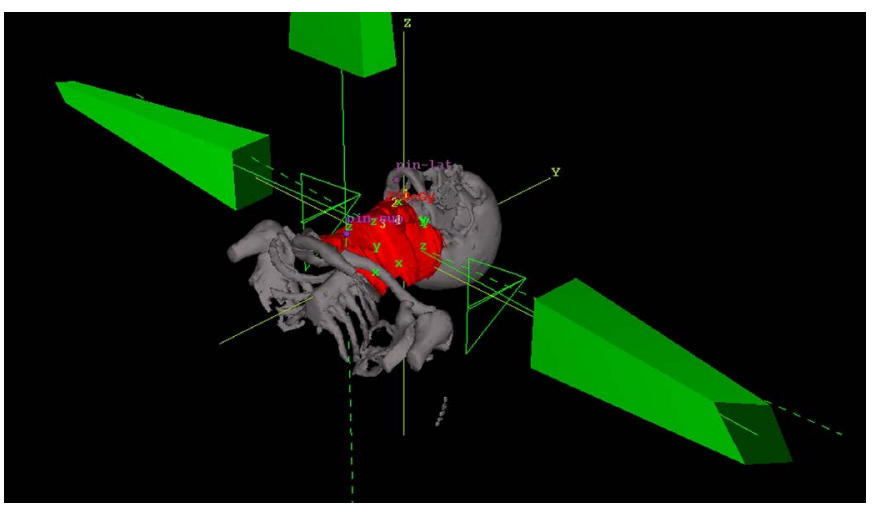

C

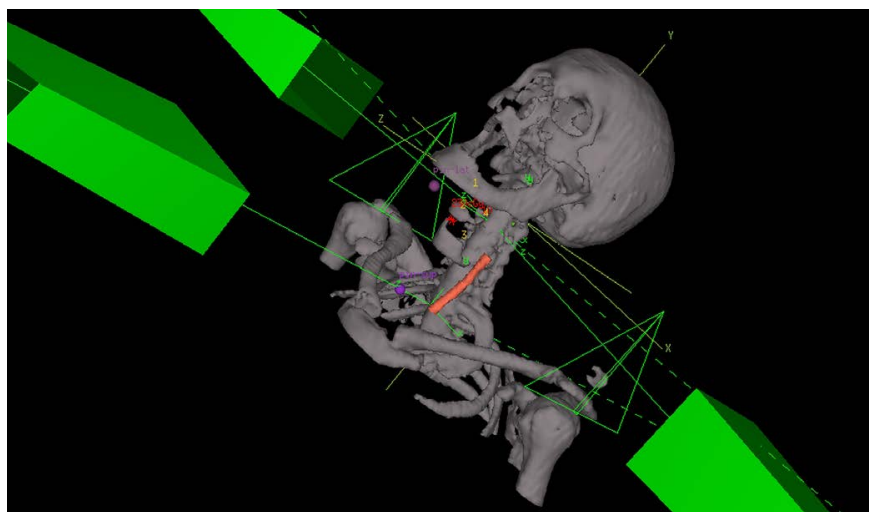

B

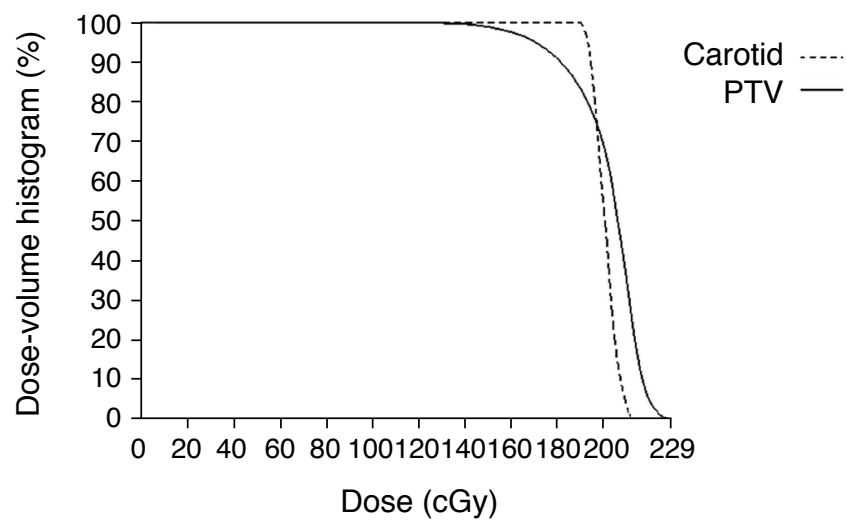

D

Fig. 1. A typical description of a three-dimensional (3D) clinical plan with contouring of the common carotid artery.

A. The common carotid artery was countered. B. The arterial volume under irradiation with three fields was reconstructed in 3D dimensions. C. A 3D view of the position of the common carotid artery by the radiation therapy volume was illustrated. D. A dose-volume histogram for the contoured common carotid artery versus the planning target volume (PTV) for one patient shows that the delivered mean dose to the common carotid artery is as high as that of the PTV.

received in each session was determined and the total mean dose was estimated based on the number of treatment sessions and the total mean dose received by the target.

\section{Ultrasonography}

All patients underwent common carotid artery ultrasound imaging before and after the completion of radiotherapy. Common carotid artery ultrasonography was performed the day before radiotherapy and 6 weeks after radiotherapy. In this study, the systolic blood pressure $\left(P_{\max }\right)$ and the diastolic blood pressure $\left(P_{\min }\right)$ were measured in the radial artery using a calibrated digital sphygmomanometer.

Physical parameters were extracted by processing the ultrasound images of the carotid artery. The subjects were placed in the supine position for at least 10 minutes before the start of imaging to bring their heart rate and blood pressure to a uniform state. B-mode imaging of the common carotid artery was performed at a distance of $2-3 \mathrm{~cm}$ from the bifurcation in the longitudinal view during three cardiac cycles at a 4.4-cm focal depth with 65 frames per second (6-11 MHz linear transducer; SSI-6000 model, Kai-Li Ultrasound, Shenzhen, China). Color Doppler imaging was also performed at a $60^{\circ}$ angle from the patient's common carotid artery to determine the peak systolic velocity $(V p)$ and the end-diastolic velocity (Ve) before and after radiotherapy. Consecutive images were recorded in the system memory for transfer to a personal computer. To perform each exam, the probe was positioned at the lateral side of the neck in the longitudinal view. Internal diameter, IMT, and longitudinal displacement of the common carotid artery were measured offline using two algorithms designed in MATLAB software (7.10.0.499, MathWorks, Natick, MA, USA) that allowed a quantitative assessment of the time rate changes from consecutive images. The maximum gradient algorithm was used to evaluate the time rate changes of internal diameter and IMT, as discussed in previous reports $[12,13]$. The block matching algorithm was also utilized to assess the instantaneous changes of longitudinal displacement of 
the arterial wall from sequential ultrasonic images. This algorithm has been described in detail elsewhere [13]. Fig. 2 shows the procedure of assessing the changes of arterial wall thickness in the radial and longitudinal directions using the maximum gradient and the block matching algorithms, respectively.

To summarize, the time-dependent movements of internal diameter, IMT, and longitudinal displacement of each vessel were determined by computational approaches (Fig. 3) to determine biomechanical behavior in response radiation-induced damage.

In Fig. 4, time-dependent changes of blood flow velocity in the common carotid artery of a patient are shown before and after radiotherapy. By recording the Doppler spectrum, blood flow velocities at peak systole $\left(\mathrm{V}_{\text {max }}\right)$, end-diastole $\left(\mathrm{V}_{\text {min }}\right)$, and mean $\left(\mathrm{V}_{\text {mean }}\right)$ were measured $(\mathrm{cm} / \mathrm{s})$.

\section{Basic Equations}

The intima-media layer is adjacent to the bloodstream, and the adventitia layer is adjacent to the tissues; this proximity causes it to move slightly. To analyze the shear elastic behavior of an organ, the shear elastic modulus was defined based on the longitudinal movements of tissue in response to a constant load. The shear elastic modulus is defined as the ratio between the shear stress and the strain caused by it [14]. The shear stress of the artery $(\tau)$ is estimated using the following equation [15]:

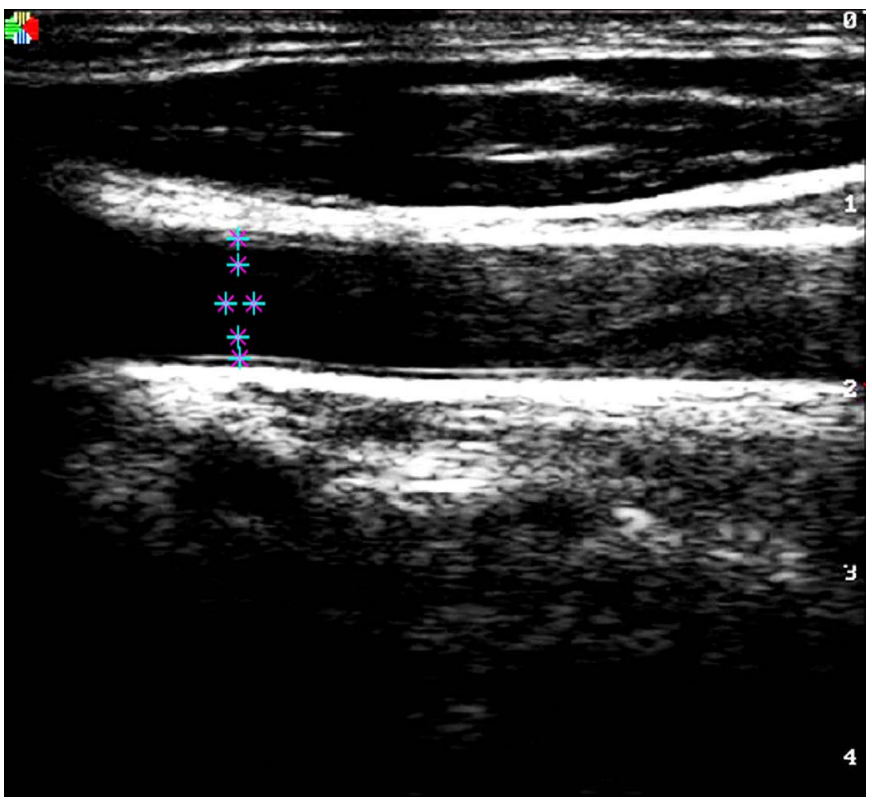

A

Fig. 2. Methodology of processing ultrasound images to estimate the arterial wall thickness movements.

A. The arterial wall was measured using the maximum gradient algorithm at the circumferential direction. B. The arterial movement was measured using the block matching algorithm at the axial direction.

e-ultrasonography.org

Ultrasonography 41(1), January 2022 

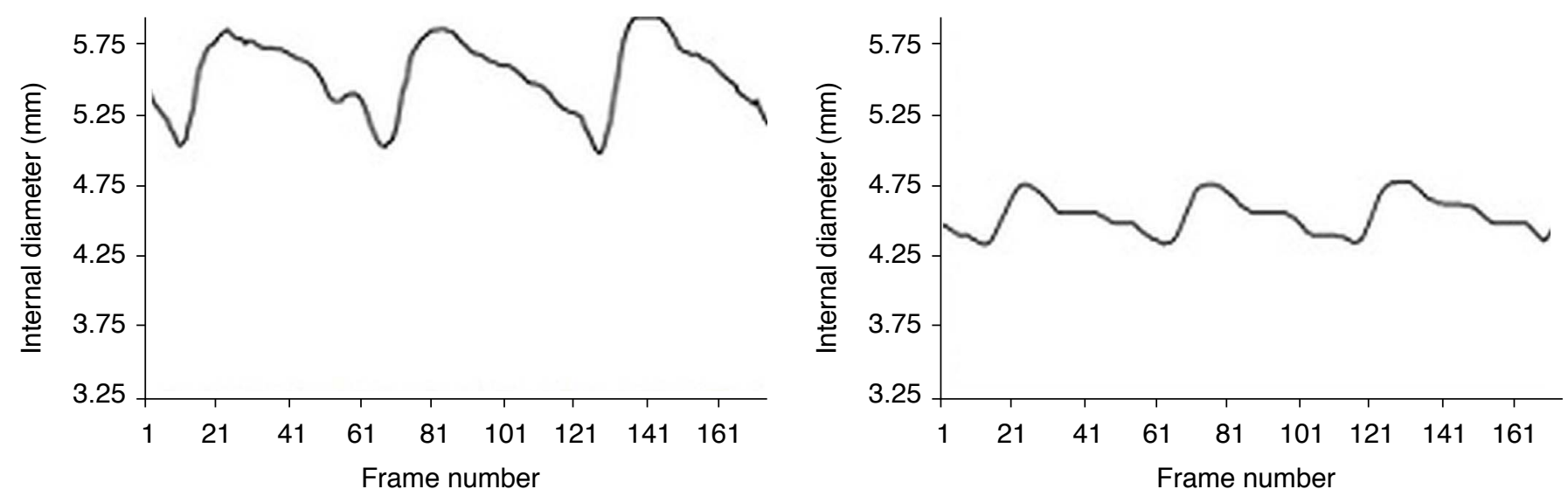

A
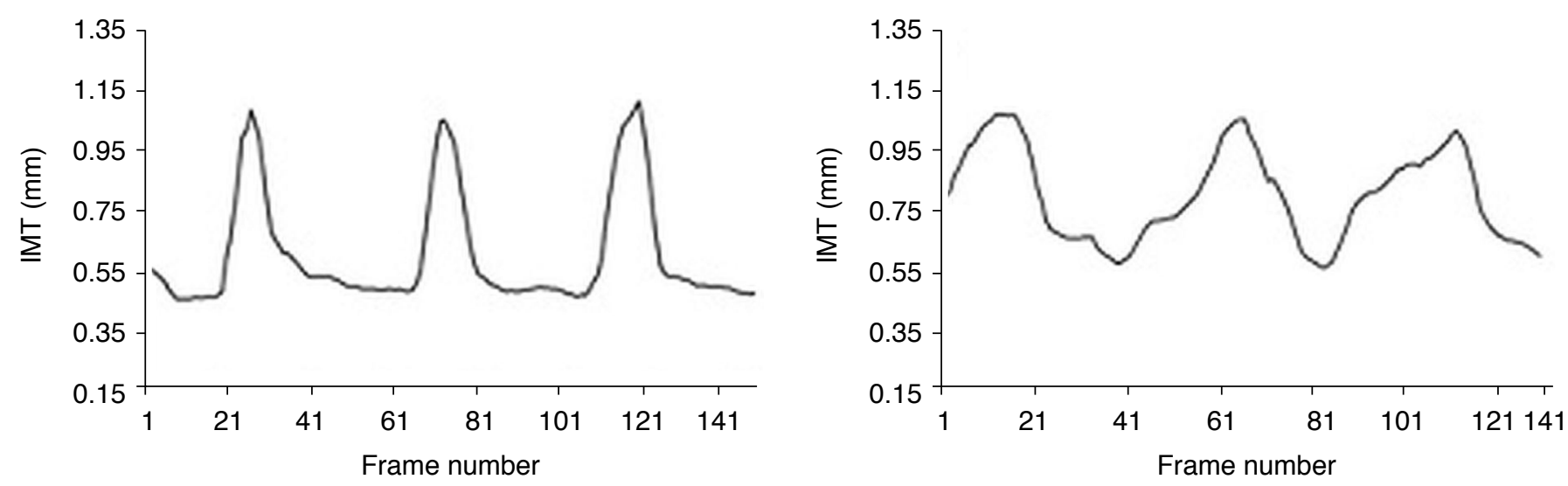

B
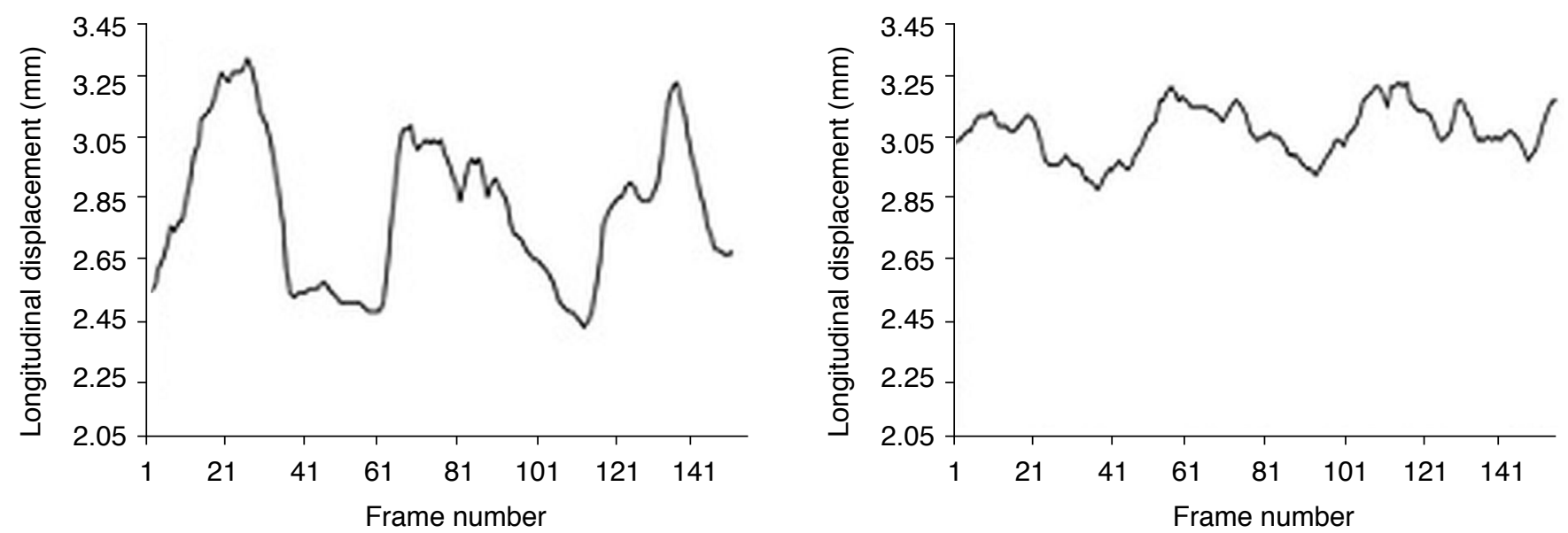

C

Fig. 3. Time-dependent changes in the common carotid artery of a patient throughout three cardiac cycles.

Internal diameter (A), intima-media thickness (IMT) (B), and longitudinal displacement (C), before (left side) and after (right side) radiotherapy are plotted. 


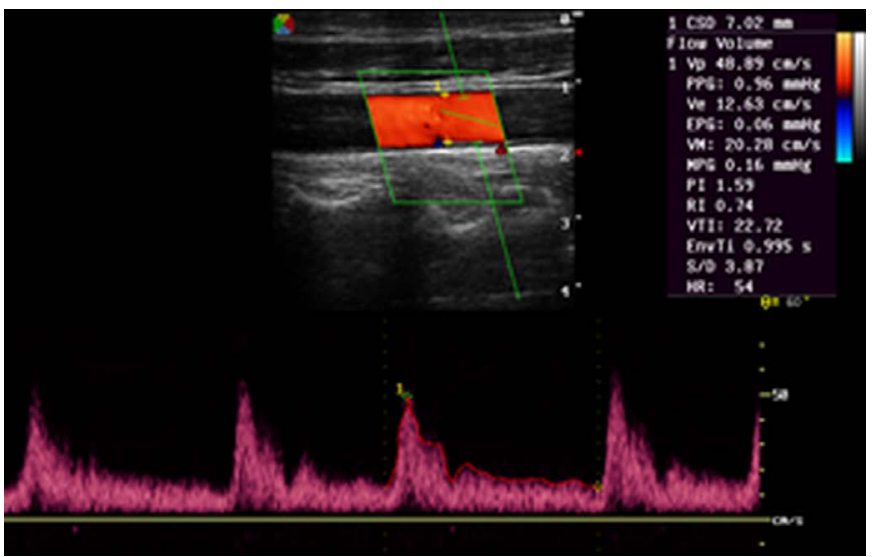

A

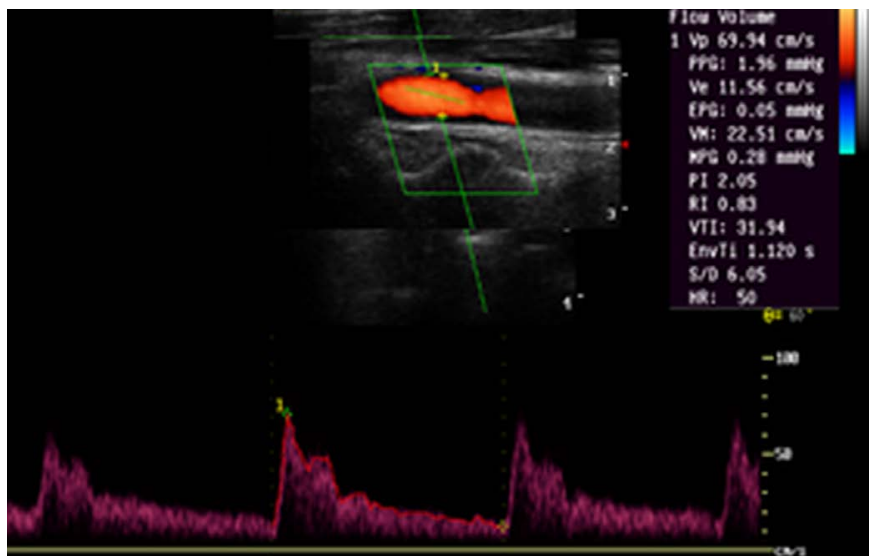

B

Fig. 4. Blood flow velocities in the common carotid artery extracted using Doppler ultrasonography, before (A) and after (B) external radiotherapy.

longitudinal movement of the common carotid artery corresponds to the difference between the maximum and minimum longitudinal displacement $\left(\Delta L=L_{\max }-L_{\min }\right)$ during the cardiac cycle $[12,17]$. Thus, the shear strain of the common carotid artery can be given simply as:

$$
\varepsilon_{\text {f }}=\operatorname{arctg}\left(\frac{\Delta L}{I M T}\right) \text {. }
$$

Therefore, the shear elastic modulus $\left(E_{s}\right)$ was calculated as the ratio between the shear stress and shear strain as:

$$
E_{S}=\frac{\left(\tau_{\max }-\tau_{\min }\right)}{\varepsilon_{l}},
$$

where $\tau_{\max }$ and $\tau_{\min }$ are the maximum and minimum shear stress quantities during the cardiac cycle, respectively.

The Young modulus of the carotid artery $(E)$ is determined by the following equation $[13,18]$ :

$$
E=\frac{\left(P_{\max }-P_{\min }\right)}{\left(\left(D_{\max }-D_{\min }\right) / D_{\min }\right)} \text {. }
$$

Pulse pressure, as the applied stress, is considered to be the difference between systolic phase pressure $\left(P_{\text {max }}\right)$ and diastolic phase pressure $\left(P_{\min }\right)$. The relative deformation of the vessel in the radial direction (radial strain) is also calculated based on the maximum systolic diameter $\left(D_{\max }\right)$ and the minimum diastolic diameter $\left(D_{\min }\right)$ of the artery in a cardiac cycle.

A schematic image of the steps of the study for each patient is shown in Fig. 5.

\section{Statistical Analysis}

In the present study, the paired-sample t test with $95 \%$ confidence intervals (with $\mathrm{P}<0.05$ interpreted as showing statistical significance) was used to compare the differences between the
Table 1. Mean dose (Gy) delivered to the PTV and CCA of head and neck cancer patients

\begin{tabular}{cccc}
\hline & PTV (Gy) & CCA (Gy) & CCA/PTV \\
\hline Dose (Gy) & $59.83 \pm 11.28$ & $49.30 \pm 15.83$ & $0.82 \pm 0.24$ \\
\hline
\end{tabular}

PTV, planning target volume; CCA, common carotid arteries.

physical and mechanical parameters of irradiated and non-irradiated arteries within the same patients (pre-and post-irradiation). The maximum sample size for the paired-sample $t$ test analysis was estimated using a confidence level of $95 \%$ and a power of $90 \%$. The statistical analysis was performed using SPSS version 21.0 (IBM Corp., Armonk, NY, USA).

\section{Results}

The mean doses received by the tumors and common carotid arteries are shown in Table 1. As demonstrated in Table 1, the mean dose received by the common carotid arteries was $17.6 \%$ lower than that received by the planning target volume. Therefore, a significant amount of the prescribed dose was received by the common carotid arteries.

The physical parameters were measured at the peak systolic and end-diastolic phases using two image processing algorithms to estimate the shear elastic modulus before and after radiotherapy (Table 2). These parameters included the peak systolic diameter $\left(D_{\max }\right)$, end-diastolic diameter $\left(D_{\min }\right)$, the blood volume flow at peak systole $\left(Q_{\max }\right)$, and end-diastole $\left(Q_{\min }\right)$, IMT, and maximum $\left(L_{\max }\right)$ and minimum longitudinal displacements of the arterial wall $\left(L_{\min }\right)$.

Treatment of the neck area with ionizing irradiation led to a 


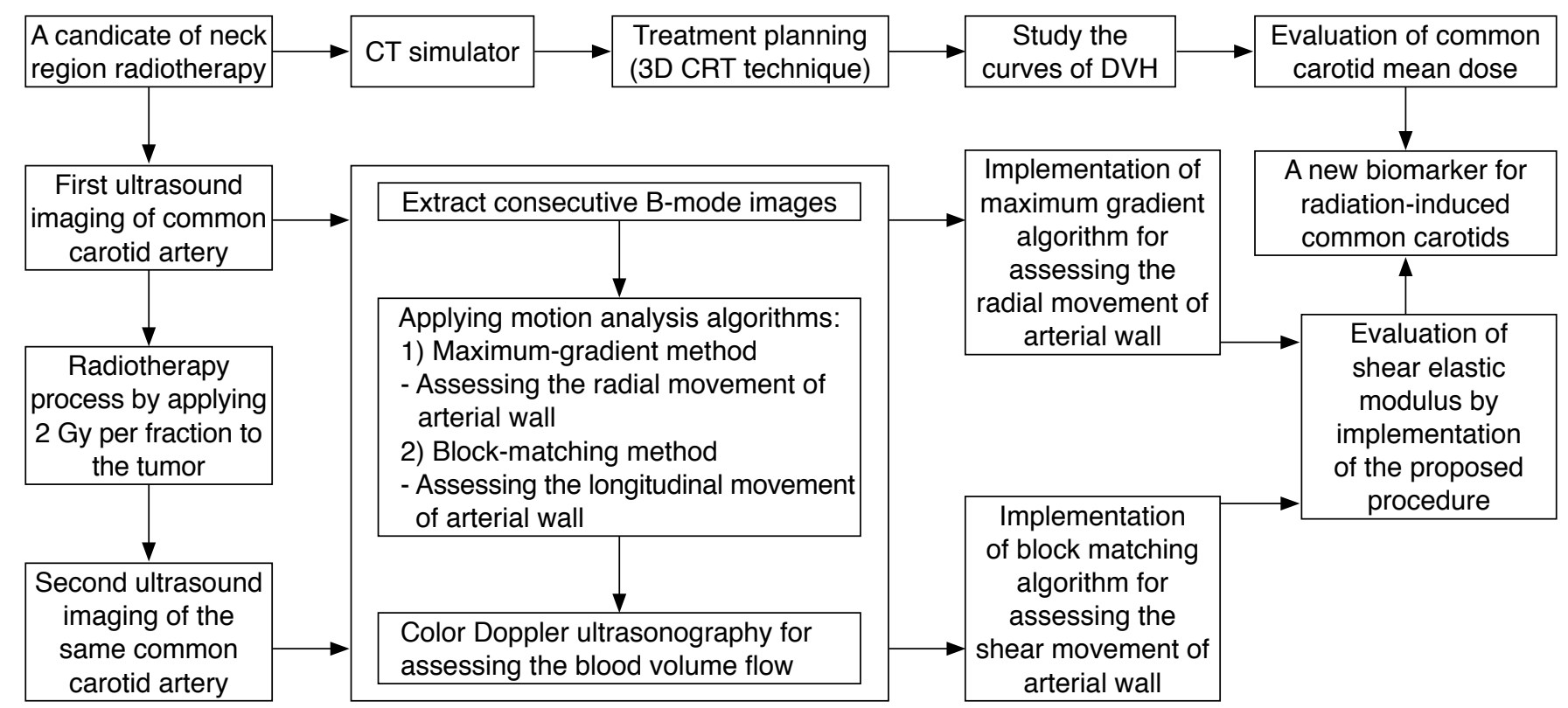

Fig. 5. Schematic diagram showing the steps to measure the shear modulus behavior of the common carotid arteries in response to external radiotherapy. CT, computed tomography; 3D CRT, three-dimensional conformal radiotherapy; DVH, dose volume histogram.

Table 2. Physical characteristics of the common carotid artery extracted using image processing algorithms before and after external radiotherapy

\begin{tabular}{lccc}
\hline \multicolumn{1}{c}{ Parameter } & Pre-irradiation & Post-irradiation & P-value \\
\hline Peak systolic diameter $(\mathrm{mm})$ & $6.42 \pm 0.67$ & $5.63 \pm 0.67$ & $<0.001$ \\
$\begin{array}{l}\text { End-diastolic diameter } \\
\text { (mm) }\end{array}$ & $5.56 \pm 0.58$ & $4.92 \pm 0.55$ & $<0.001$ \\
$\begin{array}{l}\text { Peak systolic volume flow } \\
\left(\mathrm{cm}^{3} / \mathrm{s}\right)\end{array}$ & $15.49 \pm 3.64$ & $12.97 \pm 4.44$ & 0.003 \\
$\begin{array}{l}\text { End-diastolic volume flow } \\
\left.\text { ( } \mathrm{cm}^{3} / \mathrm{s}\right)\end{array}$ & $3.13 \pm 0.88$ & $2.09 \pm 0.77$ & $<0.001$ \\
$\begin{array}{l}\text { Maximum intima-media } \\
\text { thickness (mm) }\end{array}$ & $0.80 \pm 0.14$ & $0.92 \pm 0.14$ & $<0.001$ \\
$\begin{array}{l}\text { Maximum longitudinal } \\
\text { displacement (mm) }\end{array}$ & $3.46 \pm 0.18$ & $3.54 \pm 0.15$ & $<0.001$ \\
$\begin{array}{l}\text { Minimum longitudinal } \\
\text { displacement (mm) }\end{array}$ & $2.65 \pm 0.18$ & $2.93 \pm 0.16$ & $<0.001$ \\
\hline
\end{tabular}

Values are presented as mean \pm standard deviation.

significant increase in the mean value of the IMT $(0.64 \pm 0.12$ vs. $0.56 \pm 0.09 \mathrm{~mm}, \mathrm{P}<0.001)$. The minimum values of IMT were $0.32 \pm 0.08$ and $0.37 \pm 0.09 \mathrm{~mm}$ before and after radiotherapy, respectively $(P<0.001)$. The calculated characteristics of the shear elastic modulus, including the shear stress in the peak systolic and end-diastolic phases, longitudinal stretching of the vessel, shear strain, and shear modulus, were determined before and after radiotherapy (Table 3). A significant increase was found in the shear elastic modulus following head and neck cancer treatment $(\mathrm{P}<0.001)$. The systolic blood pressure and diastolic blood pressure
Table 3. Shear stress $\left(\tau_{\max }, \tau_{\min }\right)$, longitudinal stretching of the vessel $(\Delta L)$, shear strain $\left(\varepsilon_{1}\right)$, and shear modulus $\left(E_{s}\right)$ of the common carotid artery before and after radiotherapy

\begin{tabular}{lccc}
\hline \multicolumn{1}{c}{ Parameter } & Pre-irradiation & Post-irradiation & P-value \\
\hline$\tau_{\max }(\mathrm{Pa})$ & $1.82 \pm 0.46$ & $2.28 \pm 0.69$ & 0.002 \\
$\tau_{\min }(\mathrm{Pa})$ & $0.56 \pm 0.24$ & $0.55 \pm 0.20$ & 0.821 \\
$\Delta L(\mathrm{~mm})$ & $0.81 \pm 0.25$ & $0.61 \pm 0.20$ & $<0.001$ \\
$\varepsilon_{l}($ radian $)$ & $1.04 \pm 0.36$ & $0.67 \pm 0.24$ & $<0.001$ \\
$E_{s}(\mathrm{kPa})$ & $1.38 \pm 0.98$ & $3.10 \pm 2.03$ & $<0.001$ \\
\hline
\end{tabular}

Values are presented as mean \pm standard deviation.

A P-value of $<0.05$ was considered to indicate statistical significance.

Table 4. The Young modulus and axial strain of the common carotid artery in patients before and after radiotherapy

\begin{tabular}{lccr}
\hline \multicolumn{1}{c}{ Parameter } & Pre-irradiation & Post-irradiation & P-value \\
\hline Strain (\%) & $15.74 \pm 6.17$ & $14.33 \pm 6.28$ & 0.356 \\
Young modulus (kPa) & $35.26 \pm 13.34$ & $55.36 \pm 20.96$ & $<0.001$ \\
\hline
\end{tabular}

Values are presented as mean \pm standard deviation.

of the patients were measured as $115 \pm 14 \mathrm{mmHg}$ and $78 \pm 11$ $\mathrm{mmHg}$ before radiotherapy. After radiotherapy, the corresponding values were $128 \pm 16 \mathrm{mmHg}$ and $75 \pm 10 \mathrm{mmHg}$, respectively. Systolic blood pressure significantly changed after radiotherapy $(\mathrm{P}<0.001)$.

In Table 4, the results of the mean and standard deviation of the Young modulus and axial strain in the wall of the common carotid artery in patients before and after radiotherapy are shown. 
Decreased strain after radiotherapy indicates increased arterial wall stiffness. This result confirmed our hypothesis. However, the difference was not statistically significant, which could have been due to the small sample size in this study. The Young modulus was significantly greater in the radiation-treated arteries than in the baseline measurements $(P<0.001)$.

The Young modulus and shear modulus after external irradiation were $1.57 \pm 0.59$ and $2.25 \pm 1.50$ times higher than the baseline measurements, respectively. Our findings show that arterial stiffness in the radial and longitudinal directions is a response to external radiotherapy.

\section{Discussion}

A healthy arterial wall is characterized by a normal elastic modulus in the shear and circumferential directions [19]. Therefore, local stress in the shear direction plays a particularly important role in the diagnosis of the early and long-term responses of arteries [15].

There is evidence of ischemic strokes and transient ischemic attacks due to radiation-induced atherosclerosis of the carotid artery [20]. The most well-known biomarkers of radiation-induced atherosclerosis are vascular stenosis, an increased IMT, and arterial stiffening [5]. External radiotherapy has a greater effect on the Young modulus than on other arterial biomarkers [18]. Moreover, it is thought that the short-term effects of radiation-induced disease are characterized by an inflammatory response focused on initial changes in the vascular endothelium, followed by fibrosis and necrosis in the adventitia and media layers of the carotid artery [5]. However, no report has yet investigated the axial and shear elastic moduli of the carotid artery in response to ionizing radiation.

It has been widely reported that ultrasonic characteristics are sensitive to structural changes of the common carotid artery following radiotherapy of the neck region $[5,10,18,21,22]$. Among the various biomechanical testing protocols, B-mode ultrasonography as a real-time imaging technique is a relatively convenient tool to determine the stiffness of the common carotid artery in the circumferential direction, as reflected by the Young elastic modulus [18]. Doppler ultrasound imaging also allows the measurement of blood flow parameters to determine the local shear stress of vessels [15]. In previous studies, late carotid artery damage has been reported in patients who underwent external beam radiation therapy to the neck after more than 6 months, as well as at later follow-up visits in subsequent years $[3,18]$. Significant stenosis of this artery has also been described as a late radiation injury in other reports $[1,20]$. However, how the mechanical behavior of the artery wall changes due to the acute effects of radiation is unclear, and no published study has yet investigated this issue
Extracting the radial motion of the carotid artery wall is a method to estimate the elastic modulus $[1,2,5,9]$. Recent evidence has shown that a higher value of the elastic modulus in the circumferential direction is a potentially viable biomarker of radiation-induced carotid atherosclerosis. Furthermore, the influence of external irradiation on arterial stiffness was found to be much greater than its influence on other characteristics of the vessels [18].

However, no study has yet investigated the axial displacement associated with radiation-induced atherosclerosis. Therefore, the aim of the present study was to determine the shear modulus of the common carotid arteries as a useful biomarker of radiation-induced damage. The short-term effects of ionizing radiation increased the Young modulus and shear modulus in the common carotid arteries. The arterial Young modulus and shear modulus increased by $54.01 \%$ and $124.64 \%$, respectively, after external radiotherapy. Thus, the shear elastic modulus is a more accurate biomarker for assessing radiation-induced carotid atherosclerosis.

The arterial Young modulus was reported to increase by $13.74 \%$ as a long-term effect of radiation-induced common carotid atherosclerosis [18]. Gujral et al. [18] showed that the stiffness of arteries increased after radiotherapy for at least 2 years, during which period it seemed that vascular repair mechanisms were taking place.

Recently, it has been noted that endothelial cell stiffening occurred after fractionation radiotherapy in the short term $[23,24]$. A sigmoid curve was fitted between the absorbed dose and elastic response [23]. However, there was no dose-response curve between the carotid absorbed dose and arterial stiffness in an 8-year period for patients who received head and radiotherapy [25]. Another study showed a significant increase in carotid artery stenosis after radiotherapy, but the delivered dose to the common carotid arteries after IMRT is lower than that following the 3D conformal radiotherapy [26]. Previous studies have focused on the Young elastic modulus after radiotherapy, but the shear elastic modulus has not been investigated in vessels with radiation-induced damage.

In some previous studies [5-8], the IMT of the carotid artery was reported as a reliable biomarker of late toxicity. Furthermore, as an interesting outcome, Andreassi et al. [27] reported that long-term low-dose ionizing radiation led to an increase in the IMT, 1.69\% and $5.45 \%$ for the left and right arteries, respectively, and this increase served as a useful biomarker of carotid damage. Our results show that the carotid IMT increased by $15 \%$ as an early effect of external radiotherapy (Table 2).

The shear modulus of radiation-exposed arteries was significantly higher than those of the vessels prior to radiotherapy (Table 3). This variation was associated with a significant increase in the IMT and Young modulus as well-known biomarkers of radiation-induced 
atherosclerosis (Table 2). However, the shear modulus changes were significantly different from those of any other radiation-induced biomarker, such as the lumen diameter, IMT, and blood volume flow. Therefore, the shear modulus can be introduced as the most useful biomarker to diagnose carotid stiffness following head and neck radiotherapy.

Nonetheless, a necessary condition of the present study was that a considerable mean dose was delivered to the common carotid arteries (to increase the arterial stiffness as a short-term effect). A previous report showed that the mean dose of the common carotid artery, as an at-risk organ, should be $<70$ Gy [20]. The mean absorbed dose of the common carotid arteries in this study was significant but remained within the limit. Therefore, the changes in arterial parameters resulted from the considerable mean dose received by the common carotid arteries.

The main goal of the present study was to investigate the possibility of introducing the shear modulus as a new biomarker of radiation-induced atherosclerosis. This study contained three limitations: (1) due to anatomical differences among patients, the mean absorbed dose delivered to the common carotid arteries was not exactly equal; (2) the long-term effects of irradiation have not been investigated; and (3) the small number of patients increases the magnitude of probable errors. Therefore, the long-term effects of radiotherapy on the shear behavior of the common carotid artery should be investigated in more patients.

It is clear that the biomarkers extracted by processing ultrasound images are noninvasive, safe, and easy to perform. The present study was performed to characterize the arterial shear modulus after radiotherapy as a biomarker of arterial disease. Therefore, a quantitative estimate of shear modulus for radiation-induced arteries is suggested when modern radiation therapy modalities (e.g., IMRT, radiosurgery, tomotherapy, and proton therapy) are implemented to treat cancers in the head and neck region.

External radiotherapy involving the head and neck may lead to carotid stiffening, an increased IMT, and internal diameter reduction in the short term after cancer treatment. In particular, the shear modulus is introduced as a reliable biomarker of radiation-induced carotid atherosclerosis. This study supports the hypothesis that the biomechanical properties of the arterial walls rapidly change in response to external radiotherapy.

ORCID: Alireza Mohammadkarim: https://orcid.org/0000-0003-2057-3247; Manijhe Mokhtari-Dizaji: https://orcid.org/0000-0003-1598-2038; Ali Kazemian: https:// orcid.org/0000-0002-4500-4801; Hazhir Saberi: https://orcid.org/0000-0001-7198-6207; Niloofar Ayoobi Yazdi: https://orcid.org/0000-0001-6522-7538; Mahbod Esfehani: https:// orcid.org/0000-0003-2188-0265

\section{Author Contributions}

Conceptualization: Mohammadkarim A, Mokhtari-Dizaji M, Kazemian A, Saberi H. Data acquisition: Mohammadkarim A, Mokhtari-Dizaji M, Kazemian A, Saberi H, Yazdi NA, Esfehani M. Data analysis or interpretation: Mohammadkarim A, Mokhtari-Dizaji M, Kazemian A, Saberi H. Drafting of the manuscript: Mohammadkarim A, Mokhtari-Dizaji M, Kazemian A, Saberi H, Yazdi NA, Esfehani M. Critical revision of the manuscript: Mohammadkarim A, MokhtariDizaji M, Kazemian A, Saberi H, Yazdi NA, Esfehani M. Approval of the final version of the manuscript: all authors.

\section{Conflict of Interest}

No potential conflict of interest relevant to this article was reported.

\section{Acknowledgments}

This study was approved by the Faculty of Medical Sciences, Tarbiat Modares University.

\section{References}

1. Chang YJ, Chang TC, Lee TH, Ryu SJ. Predictors of carotid artery stenosis after radiotherapy for head and neck cancers. J Vasc Surg 2009;50:280-285.

2. Zhou L, Xing P, Zou L, Shen J, Tian Y, Lu X. Middle cerebral artery stenosis in patients with nasopharyngeal carcinoma after radiotherapy: the incidence of stenosis and the risk factors. $\mathrm{Br} J$ Radiol 2016;89:20150815.

3. Kaluzny J, Kopec T, Szczepanek-Parulska E, Stangierski A, Gurgul E, Ruchala M, et al. Shear wave elastography: a new noninvasive tool to assess the intensity of fibrosis of irradiated salivary glands in head and neck cancer patients. Biomed Res Int 2014;2014:157809.

4. Vitolo V, Millender LE, Quivey JM, Yom SS, Schechter NR, JereczekFossa BA, et al. Assessment of carotid artery dose in the treatment of nasopharyngeal cancer with IMRT versus conventional radiotherapy. Radiother Oncol 2009;90:213-220.

5. Gujral DM, Shah BN, Chahal NS, Bhattacharyya S, Hooper J, Senior $\mathrm{R}$, et al. Carotid intima-medial thickness as a marker of radiationinduced carotid atherosclerosis. Radiother Oncol 2016;118:323329.

6. Gujral DM, Shah BN, Chahal NS, Senior R, Harrington KJ, Nutting CM. Clinical features of radiation-induced carotid atherosclerosis. Clin Oncol (R Coll Radiol) 2014;26:94-102.

7. Cheng SW, Ting AC, Wu LL. Ultrasonic analysis of plaque characteristics and intimal-medial thickness in radiationinduced atherosclerotic carotid arteries. Eur J Vasc Endovasc Surg 2002;24:499-504.

8. Gianicolo ME, Gianicolo EA, Tramacere F, Andreassi MG, Portaluri M. Effects of external irradiation of the neck region on intima media 
thickness of the common carotid artery. Cardiovasc Ultrasound 2010;8:8.

9. Cheng SW, Ting AC, Lam LK, Wei WI. Carotid stenosis after radiotherapy for nasopharyngeal carcinoma. Arch Otolaryngol Head Neck Surg 2000;126:517-521.

10. Mohammadkarim A, Mokhtari-Dizaji M, Kazemian A, Saberi $H$. Hemodynamic analysis of radiation-induced damage in common carotid arteries by using color Doppler ultrasonography. Ultrasonography 2018;37:43-49.

11. Garcez K, Lim CC, Whitehurst $\mathrm{P}$, Thomson D, Ho KF, Lowe M, et al. Carotid dosimetry for $\mathrm{T} 1$ glottic cancer radiotherapy. $\mathrm{Br} J$ Radiol 2014;87:20130754.

12. Soleimani $E$, Mokhtari-Dizaji $M$, Saberi H. A novel non-invasive ultrasonic method to assess total axial stress of the common carotid artery wall in healthy and atherosclerotic men. J Biomech 2015;48:1860-1867.

13. Rahmani-Cherati T, Mokhtari-Dizaji M, Vajhi A, Rostami A. Evaluation of statin therapy on endothelial function in hypercholesterolemic rabbits by automatic measurement of arterial wall movement using ultrasound images. Ultrasound Med Biol 2014;40:2415-2424.

14. Parker KJ, Doyley MM, Rubens DJ. Imaging the elastic properties of tissue: the 20 year perspective. Phys Med Biol 2011;56:R1-R29.

15. Marais L, Pernot M, Khettab H, Tanter M, Messas E, Zidi M, et al. Arterial stiffness assessment by shear wave elastography and ultrafast pulse wave imaging: comparison with reference techniques in normotensives and hypertensives. Ultrasound Med Biol 2019:45:758-772.

16. Cinthio M, Ahlgren AR, Bergkvist J, Jansson T, Persson HW, Lindstrom K. Longitudinal movements and resulting shear strain of the arterial wall. Am J Physiol Heart Circ Physiol 2006;291:H394-H402.

17. Svedlund S, Gan LM. Longitudinal common carotid artery wall motion is associated with plaque burden in man and mouse. Atherosclerosis 2011;217:120-124.

18. Gujral DM, Shah BN, Chahal NS, Bhattacharyya S, Senior R, Harrington KJ, et al. Arterial stiffness as a biomarker of radiation- induced carotid atherosclerosis. Angiology 2016;67:266-271.

19. Li Z, Du L, Wang F, Luo X. Assessment of the arterial stiffness in patients with acute ischemic stroke using longitudinal elasticity modulus measurements obtained with shear wave elastography. Med Ultrason 2016;18:182-189.

20. Fernandez-Alvarez V, Lopez F, Suarez C, Strojan P, Eisbruch A, Silver CE, et al. Radiation-induced carotid artery lesions. Strahlenther Onkol 2018;194:699-710.

21. Makita C, Okada S, Kajiura Y, Tanaka O, Asahi Y, Yamada N, et al. Vascular events from carotid artery atherosclerosis after radiation therapy for laryngeal and hypopharyngeal cancer: the incidence and risk factors. Nagoya J Med Sci 2020;82:747-761.

22. Gujral DM, Cheung WK, Shah BN, Chahal NS, Bhattacharyya $S$, Hooper J, et al. Contrast enhancement of carotid adventitial vasa vasorum as a biomarker of radiation-induced atherosclerosis. Radiother Oncol 2016;120:63-68.

23. Mohammadkarim A, Mokhtari-Dizaji M, Kazemian A, Saberi H, Khani MM, Bakhshandeh M. Dose-dependent (60)Co gammaradiation effects on human endothelial cell mechanical properties. Cell Biochem Biophys 2019;77:179-186.

24. Mohammadkarim A, Tabatabaei M, Parandakh A, Mokhtari-Dizaji M, Tafazzoli-Shadpour M, Khani MM. Radiation therapy affects the mechanical behavior of human umbilical vein endothelial cells. J Mech Behav Biomed Mater 2018;85:188-193.

25. Carpenter DJ, Mowery YM, Broadwater G, Rodrigues A, Wisdom AJ, Dorth JA, et al. The risk of carotid stenosis in head and neck cancer patients after radiation therapy. Oral Oncol 2018;80:9-15.

26. Liao W, Zhou H, Fan S, Zheng Y, Zhang B, Zhao Z, et al. Comparison of significant carotid stenosis for nasopharyngeal carcinoma between intensity-modulated radiotherapy and conventional twodimensional radiotherapy. Sci Rep 2018;8:13899.

27. Andreassi MG, Piccaluga E, Gargani L, Sabatino L, Borghini A, Faita $F$, et al. Subclinical carotid atherosclerosis and early vascular aging from long-term low-dose ionizing radiation exposure: a genetic, telomere, and vascular ultrasound study in cardiac catheterization laboratory staff. JACC Cardiovasc Interv 2015;8:616-627. 